\title{
Literature Review of the Concepts of Condylar Resorption, Incidences during Orthodontic and after Orthognathic Surgery and Treatment Measures
}

\author{
Khadeegh Alrezami ${ }^{1,2}$ and Yang $P u^{1,2, *}$ \\ ${ }^{1}$ State Key Laboratory of Oral Diseases, National Clinical Research Center for Oral Diseases, West China Hospital of Stomatology, Sichuan University, \\ Chengdu, Sichuan, China \\ ${ }^{2}$ Department of Orthodontics, West China Hospital of Stomatology, Sichuan University, Chengdu, Sichuan, China
}

\begin{abstract}
*Corresponding author: Yang Pu, State Key Laboratory of Oral Diseases, National Clinical Research Center for Oral Diseases, Department of Orthodontics, West China Hospital of Stomatology, Sichuan University, Chengdu, Sichuan, No.14, Sec. 3, Renminnan Road, Chengdu, Sichuan, 610041, China, E-mail: yangpu@scu.edu.cn
\end{abstract}

Received: 28 Apr, 2021 | Accepted: 13 May, 2021 | Published: 19 May, 2021

Citation: Alrezami K, Pu Y (2021) Literature Review of the Concepts of Condylar Resorption, Incidences during Orthodontic and after Orthognathic Surgery and Treatment Measures. Int J Dent Oral Health 7(4): dx.doi.org/10.16966/2378-7090.364

Copyright: (C) 2021 Alrezami K, et al. This is an open-access article distributed under the terms of the Creative Commons Attribution License, which permits unrestricted use, distribution, and reproduction in any medium, provided the original author and source are credited.

\begin{abstract}
Condylar resorption and its incidence at an early age or after orthognathic surgery are well-documented issues, but it is associated with high controversies regarding its etiology and management. Lack of clear understanding of its pathogenies and the limitation of the available evidence inherited for the development of a systematic treatment approach and most previous studies recommended further investigation of the problem. This review aims to cover the problem of condylar resorption and its association with orthodontic and orthognathic surgery as well as to discuss the prospective treatment measure available through the literature. According to the available literature, most previous studies covered the condylar resorption of this type of patient (skeletal class II with high mandibular plane angle) following the orthognathic surgery. However, no studies investigated the long-term condition of the condyles of the involved patient before the surgery. Furthermore, there is no evidence for the orthodontic management of this challenging condition, and most recommendations come from case reports. So, it is crucial to assess the changes that happened to the condyle during the preoperative orthodontic and correlate the finding with what happened after the surgery to provide clear evidence that may help further understand the problem of condylar resorption. This evidence could be beneficial for both patients and clinicians. CT or CBCT images have been regarded as the best choice of detailed diagnosis and investigation of condylar resorption. The incidence of condylar resorption after orthognathic surgery with or without disc repositioning of skeletal class II with high mandibular plane angle is extremely suspected. However, artificial joint replacement is considered a gold standard treatment measure in severe or relapsed cases.
\end{abstract}

Keywords: Condylar resorption; Orthognathic surgery; Orthodontic treatment; Mandibular retrusion; Remodeling

\section{Introduction}

Condylar resorption (CR) is a condition that occurs when mandibular condylar bone lysis and loss of condylar volume [1]. The term idiopathic condylar resorption (ICR) is a generic term frequently used to ascertain condyle where the specific cause is unknown. Idiopathic condylar resorption has been used to comprise several TMJ pathologic abnormalities of various origins [1,2]. Moreover, Progressive condylar resorption (PCR), as the name emphasizes, is a progressive process with the term is generally used to describe a condition that results in loss of condylar height, which can occur due to several underlying influences that are known to be associated with condylar resorption. Furthermore, condylar resorption can be classified into primary condylar resorption and secondary condylar resorption according to whether there is an exact etiology or not. The primary one could be referred to as idiopathic resorption of a progressive nature with unknown etiology. While the secondary type represents condylar resorption, which has definite local or systemic pathogenic factors $[3,4]$.
The condition of PCR is well known to have predominance in young women with ages between 15 up to 35 years and is most common in teenage girls at the pubertal growth spurt [5]. Clinically, the patients appeared with apparent high mandibular plane angles, mandibular retrognathism, and TMJ dysfunction (Figure 1) [2,6,7]. The situation is infrequent in patients with low mandibular plane angle or skeletal Class III patients $[5,8,9]$. Patients with condylar resorption commonly have associated TMJ symptoms with about $25 \%$ of patients with significant TMJ abnormality will be asymptomatic [1]. These patients are diagnostically present a challenge when undergoing orthognathic surgery since the TMJ abnormality may be unrecognized, ignored, or treated inappropriately, resulting in a poor treatment outcome with the potential redevelopment of the skeletal and occlusal deformity as further condylar resorption occurs, leading to worsening or the initiation of pain, headaches, jaw and TMJ dysfunction, and so on [1].

Many authors have been reported the occurrence of condylar resorption as being associated with orthodontic and orthognathic treatments [10-16]. However, the problem of condylar resorption 


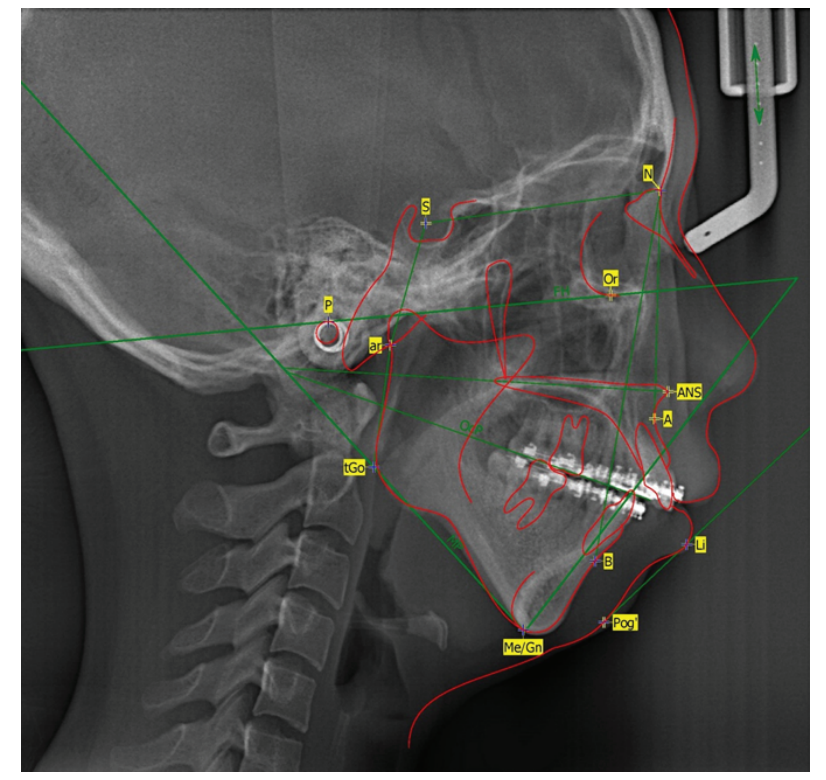

Figure 1: Adult patient with bilateral condylar resorption with characteristic of retrognathia, anterior open-bite and high mandibular plane angle.

coincidentally present during the treatment, and the treatment modalities are not the specific cause of the resorption. The condylar abnormality may have been preexisted or developed during treatment and is usually not initiated by orthodontics or orthognathic surgery. However, orthodontics and surgery can exacerbate condylar resorption [1]. The problem is considered the most controversial issue facing clinicians so forth.

\section{Etiology}

Despite the local (e.g., osteoarthritis, reactive arthritis, infection, traumatic injury) and systematic (e.g., Rheumatoid arthritis, scleroderma, systemic lupus erythematosus, and psoriatic arthritis) conditions which cause progressive condylar resorption, three main etiological theories could be associated with the idiopathic one. These theories included; sex hormone-induced necrosis, avascular necrosis caused by compression of the condyle, and resorption caused by loss of the remodeling capacity $[2,8,9]$.

Sex hormone-mediated theory; as the PCR or ICR is strongly prepossessed for teenage girls, also known as cheerleader syndrome, which could also occur during the pubertal growth phase, estrogen hormone may play a role in the aggravation of this resorption process [10]. It has been found that estrogen receptors were identified in the TMJ of patients with TMJ dysfunction [2,17-19]. This hormone is well defined to mediates the metabolism of cartilage and bone, so the increase in the estrogen receptors is thought to exaggerate response to joint loading causing synovial hyperplasia with resultant condylar resorption [2,17-19].

Avascular condylar necrosis theory; this theory advocated that pathological or mechanical compression of the vascular ligamentous retrodiscal tissue of the TMJ is suggested to lead to constriction of the small vessels. This vascular constriction leads to impairment of the circulation to the condyle with resultant asepsis condylar necrosis [20-22]. Further study believed that chronic disc displacement and a specific type of malocclusion also aggravate the impaired vascular circulation to the condyle [5]. Wolford and Cardenas have suggested that condylar resorption in patients with ICR is mediated by morphologic and secretory changes in the hypertrophic bilaminar zone of the TMJ disc [11].

Dysfunctional remodeling theory; this theory was first described by Arnett GW, et al., [14,23]. They suggested that impaired remodeling could result from increased mechanical stress of the joint or reduced joint capacity for remodeling. It was also indicated that age and systemic and hormonal factors could inversely affect this process $[8,14,23,24]$. Several studies urged that condylar resorption has occurred after increased mechanical loading of the TMJ following orthodontic treatment, orthognathic surgery, trauma, internal derangement, occlusal therapy, or parafunctional habits [8,11,14,23-27].

\section{Predisposing and risk factors for condylar resorption}

The risk factors can be categorized into two parts: patient-related or surgery-related risk factors. Patient risk factors include age, gender, physiology, systemic disorders, medications, bone density, dental occlusion, and mandibular anatomy $[2,11,28]$. Among the surgery population, young females with a skeletal class II malocclusion, mandibular retrognathism, high or wide mandibular plane angles, a low posterior-to-anterior facial height ratio, and posteriorly inclined condylar neck are known to be at an increased risk for condylar resorption $[2,4,11,28]$.

It has been found that ICR occurs with a female to male ratio of 9:1 with an age range between 10-40 years, but the greatest incidence is during pubertal growth $[2,11]$. Wolford LM, et al., reported that it rarely happens after the age of 15 years $[1,10]$. Patients with progressive condylar resorption often have a dolichocephalic facial type with high occlusal and mandibular plane angles. However, the condition is infrequent with those with brachycephalic facial type $[2,5,9]$.

Patients are usually seen with skeletal class II occlusion with an anterior open bite; however, it is rare in patients with skeletal class III [2]. Furthermore, patients with a low posterior-to-anterior facial height ratio, and posteriorly inclined condylar neck are susceptible to be at high risk for condylar resorption $[2,4,11,28]$. Orthognathic surgery and its associated condylar displacement or surgical changes are claimed to be a main predisposing factor of progressive condylar resorption in patients with high occlusal and mandibular plane angles or with low posterior-to-anterior facial height ratio, and posteriorly inclined condylar neck. This issue has been profusely covered in the previous studies; however, the issue still highly doubtful [3].

A previous study claimed that condyles with preexisting signs of osteoarthritis might be at risk for progressive resorption [28]. O'Ryan $\mathrm{F}$ and Epker BN [29] assessed the morphologic changes of the condyle and considered the radiographic features in patients presenting for orthognathic surgery. The authors reported less dense and less discreetly oriented trabeculae in patients with a high mandibular plane angle than patients with a low mandibular plane angle when they assessed bone density and orientation of the trabeculae of the condyles [29]. Also, it was noted that patients with a steep mandibular plane tended to have smaller condyles compared with those with a flat mandibular plane. The ratio of cortical to the cancellous bone in teenagers and adolescents is less than that in adults. This may be one reason for increased susceptibility to condylar resorption and remodeling in young teenagers [30]. Surgery-related factors included the magnitude of surgical mandibular movement, postsurgical condylar displacement, and the fixation method [28]. The literature involving the surgical-related factors will be discussed later in detail. 


\section{Diagnosis tools and considerations}

The diagnosis of the problem is mainly based on the anamnesis, clinical and radiographic investigations. Previous clinical records such as intraoral and extraoral photos and dental casts can also be used to investigate the problem. Analysis of the serial photographic records, dental models, or lateral cephalograms can help determine the disease activity and progression $[1,10,11]$. Radiological imaging plays the primary role in diagnosing condylar resorption. Traditionally, the diagnosis has depended on conventional two-dimensional images such as orthopantomogram and lateral cephalogram that show changes in condylar height and shape.

Computed tomography $(\mathrm{CT})$ is required in advanced cases with clear signs in the previously mentioned imaging. During the patient's evaluation with suspected condylar resorption based on changes in the occlusion or relapse after orthodontic and orthognathic treatment or TMJ dysfunction, advanced imaging and hematological investigations are required to establish the diagnosis [28]. A panoramic radiograph is useful for screening the changes in the shape of the condylar surface and mandibular ramus height. Cone-beam computed tomography can be used in patients with a high suspicion of condylar resorption to provide accurate information regarding the size, shape, quality, volume and relationship of the bony components of the TMJ [31-33]. In CT or $\mathrm{CBCT}$, the condyle will appear with the disappearance of the cortical layer, irregularity of the condylar surface, and subcortical microcyst formation [34]. Furthermore, Hatcher DC, et al., [35] reported that ICR proceeds through a destructive phase of loss of the cortex that begins on the anterosuperior condylar surface. Then cavitation extends into subchondral bone, resulting in loss of condylar volume. This active phase is followed by the reparative phase of condylar flattening and surface recortication.

Magnetic resonance imaging (MRI) is also useful, particularly to assess the articular disc. Recently, it has been published that specific TMJ MRI findings for synovial diseases and ICR, and it is expected that MRI examination will likely play an essential role in the diagnosis and management of patients with TMJ condylar resorption [36].

Bone scans or nuclear imaging using skeletal scintigraphy with quantifiable TC 99 scans (technetium-99m) with single-photon emission computed tomography (SPECT) can detect the activity of the disease. However, it cannot differentiate condylar resorption activity from other inflammatory and hyperactivity conditions. The interpretation of quantitative measurements of the radioisotope uptake also may vary from the inflammatory and hyperactivity conditions as well as from side to side in growing patients [37-39].

\section{Treatment strategies of patients with condylar resorption}

The ideal treatment of ICR remains controversial, with the most challenging aspect of treatment that faces the clinician is the difficulty of assessing whether condylar resorption is active or not. Condylar resorption continues in most individuals for a variable period, and its duration is unpredictable $[2,9,28]$. The treatment ranges from a conservative approach (such as occlusal splint therapy, medicine, orthodontic treatment, or restorative therapy) to a surgical approach [3]. It has been claimed that the definitive treatment that involves occlusal or skeletal alteration should be delayed until there is sufficient evidence of resorption cessation. The successful treatment depends on the achievement of optimal esthetic and functional outcomes and the results' stability.

A. Conservative treatment: It has been proposed to start the splint therapy as soon as possible when the resorption sign started and may be used with or without the concurrent orthodontic treatment. It has been suggested to unload the condyle to relieve the discomfort and muscular pain. However, the evidence was not sufficient within the literature since most of the study deals with the surgical approach [8]. A recent systematic review by $\mathrm{He} \mathrm{Z}$, et al., [3] stated that conservative management usually includes occlusal splints, medicine, orthodontic and restorative treatment. Several studies chose conservative treatment alone to control CR in progression and it might come to a stable result. However, the conservative treatment may arrest the resorption, but the dentofacial deformities due to CR need further correction.

In the systematic review of Sansara K, et al., [8] only two studies with small sample sizes were included. The two studies reported management with conservative management of ICR using occlusal splints with or without orthodontic treatment. These two studies included only 17 patients and declaring that the patients treated by occlusal splints with or without orthodontic got a functional occlusion and tolerable temporomandibular joint complaints; however, the relapses were not reported [40,41]. Kuroda S, et al., [16] reported stable long-term treatment results following conservative splints and active orthodontic therapy in case of severe condylar resorption with rheumatoid arthritis.

Relatively successfully pharmacological management of rheumatoid arthritis or osteoarthritis has been reported $[8,42]$. However, its application in the management of ICR is still unemployed throughout the literature. So, it is essential to consider that the pathogenesis of resorption in ICR may differ from rheumatoid arthritis or osteoarthritis [8]. Consequently, further studies to evaluate the efficacy and outcome of non-surgical and pharmacological approaches of ICR are warranted.

B. Surgical treatment: The appropriate treatment of the patient with condylar resorption is still highly controversial, with the most challenging part is to define the activity of the condylar resorption. Condylar resorption can continue in most patients for a variable period, and its duration is inconstant. Papadaki ME, et al., [9] reported that condylar resorption could become inactive after one to five years, but the resorptive process may be reactivated later. So, it is important to differentiate between active and inactive condylar resorption. The authors also stated that the resorptive process rarely continues beyond 40 years of age. Nevertheless, Wolford LM, et al., reported that it rarely happens after the age of 15 years $[1,10]$.

The surgical intervention of patients with condylar resorption is mainly based on the type of the resorption. It is important to exclude the known local and systemic causes of condylar resorption (e.g., autoimmune/connective tissue diseases, hormonal, metabolic disturbances, trauma, previous TMJ surgery or pathology) by serial clinical examinations, diagnostic imaging, laboratory investigations, and consultation with a rheumatologist [28]. A recent systematic review urged that the management of condylar resorption before or after orthognathic surgery have to be formulated depending on the severity of resorption [3]. The available surgical treatment options ranging between TMJ disc repositioning as a sole treatment, TMJ disc repositioning with concurrent orthognathic surgery, orthognathic surgery alone, or condylectomy with autologous/alloplastic joint reconstruction $[1,2,5,11,28]$ (Figure 2).

According to Wolford and Cardenas protocol, they proposed that patients with condylar resorption have concomitant TMJ anterior disc displacement and orthognathic treatments [11]. So, they suggested the treatment be formulated based on the condition of the disc. If the disc is salvageable, excision of the synovial tissue and disc repositioning with Mitek anchor followed by orthognathic correction 


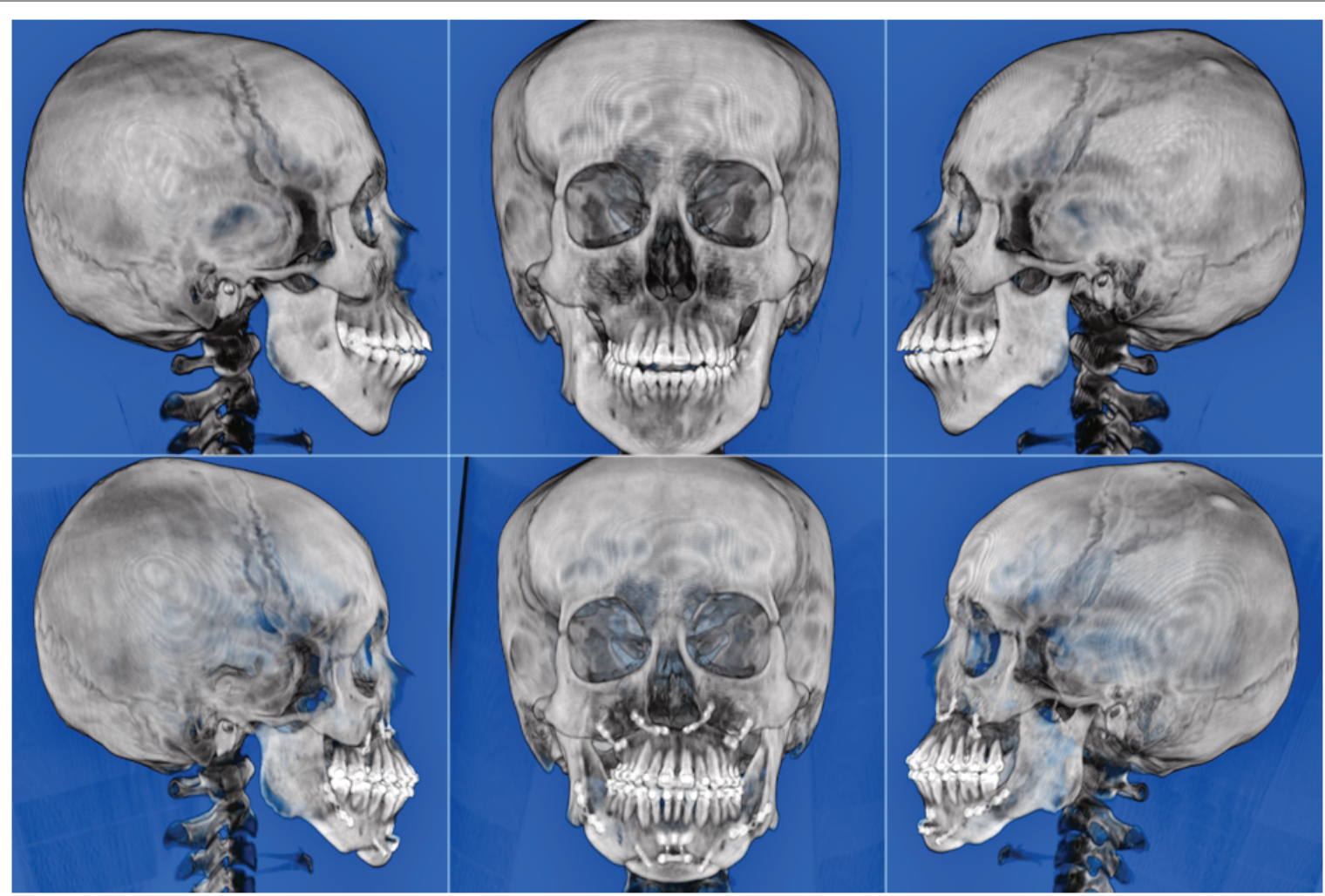

Figure 2: Shows the treatment of adult female complaining of condylar resorption and mandibular retrognathia using bimaxillary orthognathic surgery-Le Fort I osteotomy, bilateral sagittal spilt osteotomy, and genioplasty.

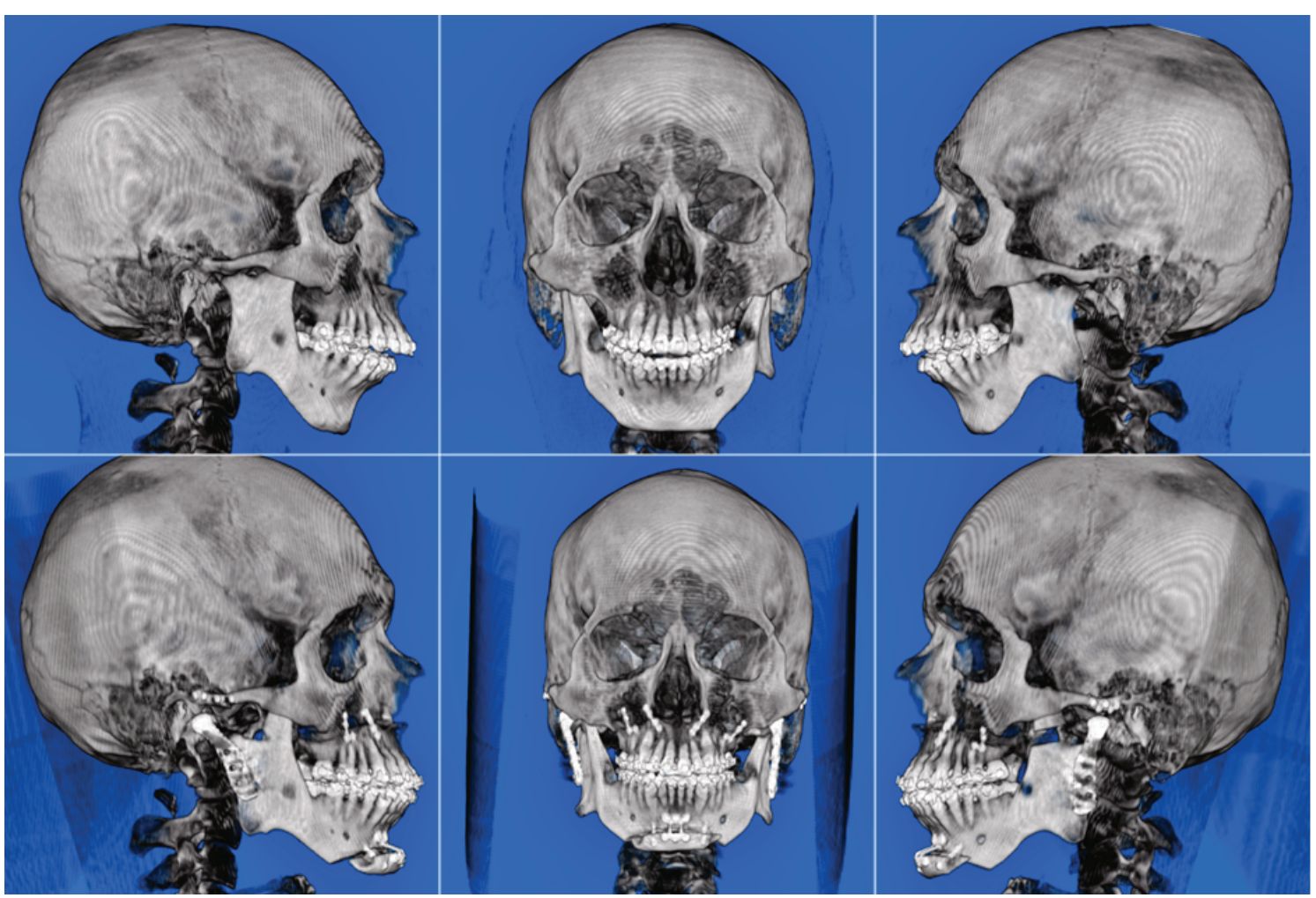

Figure 3: Shows the treatment of adult male complaining of condylar resorption and mandibular retrognathia using Le Fort I osteotomy, bilateral artificial joints, and genioplasty. 
of the jaw and occlusal deformities are recommended [11]. If the disc is not salvageable but the condyle height still adequate, then it is recommended to replace the disc with autogenous tissue. If the disc and condyle were not salvageable, it is recommended to replace the whole joint with artificial joints as a gold standard measure or autogenous tissue and bone graft (costochondral or sternoclavicular graft) (Figure 3) [11]. The authors reported that no relapse was experienced over 18 months to 12 years. Goncalves JR, et al., [43] and Gomes LR, et al., [44] reported that orthognathic surgery with simultaneous disc repositioning decreases condylar resorption incidence following the orthognathic surgery. In contrast, several authors deal with this type of patients using orthognathic surgery only and reporting an uneven amount of progressive resorption and relapse [26,45-48]. Furthermore, Bianchi J, et al., [49] reported no significant differences in skeletal stability between the patients treated by maxillomandibular advancement surgeries with disc repositioning versus those without disc repositioning.

\section{D Condylar resorption during orthodontic and orthognathic surgery}

Condylar remodeling is defined as a physiological balance between bone resorption and the bone formation process of the condyle to be adapted to the new functional requirements of the TMJ. When these functional or traumatic loads exceed the natural adaptive capacity of the condyle, bone resorption occurs. Condylar resorption is regarded as a well-documented but poorly understood pathological entity, which shows an irreversible progressive alteration of condylar morphology, as well as reduction of condylar volume [3]. Several studies dealing with condylar remodeling, particularly resorption following orthognathic surgery of patients with skeletal class II occlusion [12,31,43-47].

A case report of Kuroda S, et al., included one patient with mandibular retrognathism and anterior open bite combined with severe condylar resorption as a result of rheumatoid arthritis. The patient was treated by splint therapy and active orthodontic treatment. They reported those five years after the treatment, the occlusion remained stable without further condylar resorption. The previous study just included one patient and used a two-dimensional radiograph for the evaluation [16]. Park JH, et al., [50] reported a case (12-year-old female) diagnosed with ICR 10 months after orthodontic treatment completion of skeletal class II division 1 malocclusion. They concluded that orthodontists have to closely monitor the patients and offer detailed treatment options to patients with risk factors for ICR that might develop at any stage of orthodontic treatment. Lee GH, et al., [51] case report of a patient with skeletal class II open bite and progressive condylar resorption and stated that TMJ stabilization with splint therapy should precede the orthodontic or orthognathic treatment. They claimed that this procedure resulted in stable occlusal results without relapse over two years of follow-up.

Exposto CR, et al., [13] included 16 patients with a mean age of 15 years old suffering from ICR subjected to orthodontic treatment and found a decrease in the condylar height of average $0.8 \mathrm{~mm}$ with $75 \%$ of the condyles' radiological appearance did not change during the 2-year follow-up period. The authors also reported that during observation, the radiological appearance of some condyles improved from an erosive and repair state to a healthy state [13].

Bilateral sagittal split ramus osteotomy and Le Fort I osteotomy was the main orthognathic treatment techniques used to treat patients with mandibular retrognathism with a high mandibular plane angle [12,31,43-47]. The following studies investigated condylar remodeling or resorption included patients treated mainly by bilateral sagittal split ramus osteotomy and Le Fort I osteotomy. Kobayashi T, et al., [52] investigated the condylar resorption following mandibular advancement surgery of 6 patients with a sign of condylar resorption treated by bilateral sagittal split ramus osteotomy and Le Fort I osteotomy. They found that patients with clockwise rotation of the mandible and retrognathism in preoperative cephalograms; or with a radiographic sign of condyle resorption on preoperative CT treated by wide mandibular advancement and counterclockwise rotation of the mandible seemed to be at risk of condylar resorption postoperatively. They concluded that the mandible should be operated surgically only when the condyles are stable on radiographs [52].

Goncalves JR, et al., [43] three-dimensionally evaluated the condylar remodeling in 27 patients with skeletal class II with high mandibular plane angle. They compare bimaxillary surgical advancement with or without articular disc repositioning with one year follow-up period. They reported that more than half the patients in both groups experienced condylar resorption of at least $1.5 \mathrm{~mm}$ one year after surgery. Also, they reported that bone apposition was observed only in the group treated by disc repositioning and orthognathic surgery (superior surface in $26.4 \%$, the anterior surface in $23.4 \%$, the posterior surface in $29.4 \%$, the medial surface in $5.9 \%$, and the lateral surface in $38.2 \%)[43]$.

Xi T, et al., [45] included a total of 56 patients with mandibular retrusion to evaluate the $3 \mathrm{D}$ volumetric changes following mandibular advancement by BSSRO. They reported that condylar resorption was significantly correlated with skeletal relapse, with condylar volume loss of greater than $17 \%$ of the original volume exhibited relapse similar to that happened with PCR. Moreover, the author found that females with a high mandibular angle who showed postoperative condylar resorption were particularly at risk for long-term relapse. Their study also claimed suggested that gender, preoperative condylar volume, and improvement of mandibular plane angle were prognostic factors for condylar resorption [45].

Later, Xi T, et al., [53] also investigated the role of condylar rotation on the long-term mandibular relapse using the same population of the previous study. They reported that gender, preoperative condylar volume, postoperative condylar remodeling, counterclockwise rotation of the proximal segment, and the amount of surgical advancement were prognostic factors for skeletal relapse. However, the role of the magnitude of the mandibular plane angle in relapse is questionable. Further study of the same author claimed that young female patients who underwent large maxillomandibular advancement and postoperative decreased condylar volume (resorption) were particularly at risk for skeletal relapse [46].

Gomes LR, et al., [44] investigated the risk factors predisposing to condylar resorption and remodeling and overall volumetric changes of skeletal class II patients with high mandibular plane angle following counterclockwise rotation of the maxillomandibular complex. They found older patients were more susceptible to overall condylar volume reduction. Moreover, statistically significant correlations were detected between condylar remodeling changes in the follow-up period and presurgical facial characteristics, the magnitude of the surgical procedure, and condylar displacement changes. They concluded that postsurgical positional changes were only weakly associated with the remodeling in the follow-up period; thereby suggesting that other risk factors may play a role in condylar resorption [44].

Regarding the stability of the surgical correction of skeletal class II patients with high mandibular plane angle by counterclockwise rotation of the maxillomandibular complex, Yin Q, et al., [47] 
quantitatively assessed the correlation between the condylar resorption and skeletal relapse following mandibular advancement in skeletal class II malocclusion patients. They found a moderately significant correlation between condylar height alteration and postoperative mandibular relapse of more than $1 \mathrm{~mm}$. Gomes LR, et al., [54] quantitatively assessed condylar displacement following counterclockwise maxillomandibular advancement surgery with or without articular disc repositioning and their impacts on the longterm surgical stability. They concluded that counterclockwise rotation of the maxillomandibular complex of patients with healthy joint or simultaneous disc repositioning surgery shows stable postsurgical outcomes. However, Bianchi J, et al., stated no significant difference in skeletal stability between the patients subjected to TMJ disc repositioning followed by orthognathic surgery compared to those undergone orthognathic surgery alone [49].

Regarding the effect of preoperative higher mandibular plane angle on the incidence of condylar resorption, this issue is still in doubt. Gomes LR, et al., [44] urged that higher preoperative mandibular plane angle is not associated with increased condylar resorption and overall condylar volume reduction post-surgically. Hoppenreijs TJ, et al., [40] insisted that the mandibular plane angle is less critical in postoperative condylar resorption incidence since the resorption could be noticed in patients with an open bite and deep bite. Xi T, et al., [45] also reported that surgical relapse was not correlated with the preoperative mandibular plane angle. These authors assumed that patients with high mandibular plane angles have smaller condyles prone to the counterclockwise rotation with a large average of displacement during the surgery $[40,45]$.

\section{Conclusion}

According to the available literature, most previous studies covered the condylar resorption of this type of patient (mandibular retrognathism with high mandibular plane angle) following orthognathic surgery. However, no studies investigated the long-term condition of the condyles of the involved patient before the surgery. Furthermore, there is no evidence for the orthodontic management of this challenging condition, and most recommendations come from case reports. So, it is crucial to assess the changes that happened to the condyle during the preoperative orthodontic and correlate the finding with what happened after the surgery to provide clear evidence that may help further understand the problem of condylar resorption. This evidence could be beneficial for both patients and clinicians.

CT or CBCT images have been regarded as the best choice of detailed diagnosis and investigation of condylar resorption. The incidence of condylar resorption after orthognathic surgery with or without disc repositioning of skeletal class II with high mandibular plane angle is extremely suspected. However, artificial joint replacement is considered a gold standard treatment measure in severe or relapsed cases.

\section{Funding}

None

\section{References}

1. Wolford LM, Gonçalves JR (2015) Condylar resorption of the temporomandibular joint: how do we treat it? Oral Maxillofac Surg Clin North Am 27: 47-67.

2. Mitsimponas K, Mehmet S, Kennedy R, Shakib K (2018) Idiopathic condylar resorption. Br J Oral Maxillofac Surg 56: 249-255.
3. He Z, Ji H, Du W, Xu C, Luo E (2019) Management of condylar resorption before or after orthognathic surgery: A systematic review. J Craniomaxillofac Surg 47: 1007-1014.

4. Hwang SJ, Haers PE, Seifert B, Sailer HF (2004) Non-surgical risk factors for condylar resorption after orthognathic surgery. J Craniomaxillofac Surg 32: 103-111.

5. Posnick JC, Fantuzzo JJ (2007) Idiopathic condylar resorption: current clinical perspectives. J Oral Maxillofac Surg 65: 1617-1623.

6. Kerstens HC, Tuinzing DB, Golding RP, der Kwast WAV (1990) Condylar atrophy and osteoarthrosis after bimaxillary surgery. Oral Surg Oral Med Oral Pathol 69: 274-280.

7. Moore KE, Gooris PJ, Stoelinga PJ (1991) The contributing role of condylar resorption to skeletal relapse following mandibular advancement surgery: report of five cases. J Oral Maxillofac Surg 49: 448-460.

8. Sansare K, Raghav M, Mallya SM, Karjodkar F (2015) Managementrelated outcomes and radiographic findings of idiopathic condylar resorption: a systematic review. Int J Oral Maxillofac Surg 44: 209216.

9. Papadaki ME, Tayebaty F, Kaban LB, Troulis MJ (2007) Condylar resorption. Oral Maxillofac Surg Clin North Am 19: 223-234.

10. Wolford LM (2001) Idiopathic condylar resorption of the temporomandibular joint in teenage girls (cheerleaders syndrome). Proc (Bayl Univ Med Cent) 14: 246-252.

11. Wolford LM, Cardenas L (1999) Idiopathic condylar resorption: diagnosis, treatment protocol, and outcomes. Am J Orthod Dentofacial Orthop 116: 667-677.

12. Gonçalves JR, Cassano DS, Wolford LM, Santos-Pinto A, Márquez IM (2008) Postsurgical stability of counterclockwise maxillomandibular advancement surgery: affect of articular disc repositioning. J Oral Maxillofac Surg 66: 724-738.

13. Exposto $C R$, Stoustrup $P$, Kristensen KD, Dalstra $D$, Pedersen TK (2020) Condylar changes in patients with idiopathic condylar resorption: retrospective 2-year follow-up CBCT-based case-control study. Eur J Orthod.

14. Arnett GW, Milam SB, Gottesman L (1996) Progressive mandibular retrusion-idiopathic condylar resorption. Part II. Am J Orthod Dentofacial Orthop 110: 117-127.

15. Kristensen KD, Schmidt B, Stoustrup P, Pedersen TK (2017) Idiopathic condylar resorptions: 3-dimensional condylar bony deformation, signs and symptoms. Am J Orthod Dentofacial Orthop 152: 214-223.

16. Kuroda S, Kuroda Y, Tomita Y, Tanaka E (2012) Long-term stability of conservative orthodontic treatment in a patient with rheumatoid arthritis and severe condylar resorption. Am J Orthod Dentofacial Orthop 141: 352-362.

17. Abubaker AO, Raslan WF, Sotereanos GC (1993) Estrogen and progesterone receptors in temporomandibular joint discs of symptomatic and asymptomatic persons: a preliminary study. J Oral Maxillofac Surg 51: 1096-100.

18. Milam SB, Aufdemorte TB, Sheridan PJ, Triplett RG, Van Sickels JE, et al. (1987) Sexual dimorphism in the distribution of estrogen receptors in the temporomandibular joint complex of the baboon. Oral Surg Oral Med Oral Pathol 64: 527-532.

19. Tsai CL, Liu TK, Chen TJ (1992) Estrogen and osteoarthritis: A study of synovial estradiol and estradiol receptor binding in human osteoarthritic knees. Biochem Biophys Res Commun 183: 12871291. 
20. Chuong R, Piper MA (1993) Avascular necrosis of the mandibular condyle-pathogenesis and concepts of management. Oral Surg Oral Med Oral Pathol 75: 428-432.

21. Chuong R, Piper MA, Boland TJ (1995) Osteonecrosis of the mandibular condyle. Pathophysiology and core decompression. Oral Surg Oral Med Oral Pathol Oral Radiol Endod 79: 539-545.

22. Schellhas KP, Wilkes CH, Fritts HM, Omlie MR, Lagrotteria LB (1989) $M R$ of osteochondritis dissecans and avascular necrosis of the mandibular condyle. AJR Am J Roentgenol 152: 551-560.

23. Arnett GW, Milam SB, Gottesman L (1996) Progressive mandibular retrusion-Idiopathic condylar resorption. Part I. Am J Orthod Dentofacial Orthop 110: 8-15.

24. Mercuri LG (2008) Osteoarthritis, Osteoarthrosis, and Idiopathic Condylar Resorption. Oral Maxillofac Surg Clin North Am 20: 169183.

25. Bouwman JP, Kerstens HC, Tuinzing DB (1994) Condylar resorption in orthognathic surgery: The role of intermaxillary fixation. Oral Surg Oral Med Oral Pathol 78: 138-141.

26. Sesenna E, Raffaini M (1985) Bilateral condylar atrophy after combined osteotomy for correction of mandibular retrusion a case report. J Maxillofac Surg 13: 263-266.

27. Tanaka E, Detamore MS, Mercuri LG (2008) Degenerative Disorders of the Temporomandibular Joint: Etiology, Diagnosis, and Treatment. J Dent Res 87: 296-307.

28. Chigurupati R, Mehra P (2018) Surgical Management of Idiopathic Condylar Resorption: Orthognathic Surgery versus Temporomandibular Total Joint Replacement. Oral Maxillofac Surg Clin North Am 30: 355-367.

29. O'Ryan F, Epker BN (1984) Temporomandibular joint function and morphology: Observations on the spectra of normalcy. Oral Surg Oral Med Oral Pathol 58: 272-279.

30. Hoppenreijs TJM, Maal T, Xi T (2013) Evaluation of Condylar Resorption Before and After Orthognathic Surgery. Semin Orthod 19: 106-115.

31. Hoppenreijs TJM, Freihofer HPM, Stoelinga PJW, Tuinzing DB, van't Hof MA (1998) Condylar remodelling and resorption after Le Fort and bimaxillary osteotomies in patients with anterior open bite: $A$ clinical and radiological study aesthetic and reconstructive surgery. Int J Oral Maxillofac Surg 27: 81-91.

32. Gomes LR, Gomes MR, Gonçalves JR, Ruellas AC, Wolford LM, et al. (2016) Cone beam computed tomography-based models versus multislice spiral computed tomography-based models for assessing condylar morphology. Oral Surg Oral Med Oral Pathol Oral Radiol 121: $96-105$

33. Gomes LR, Gomes M, Jung B, Paniagua B, Ruellas AC, et al. (2015) Diagnostic index of three-dimensional osteoarthritic changes in temporomandibular joint condylar morphology. J Med Imaging (Bellingham) 2: 034501

34. Borstlap WA, Stoelinga PJW, Hoppenreijs TJM, van't Hof MA (2004) Stabilisation of sagittal split advancement osteotomies with miniplates: a prospective, multicentre study with two-year follow-up: Part III-Condylar remodelling and resorption. Int J Oral Maxillofac Surg 33: 649-655.

35. Hatcher DC (2013) Progressive Condylar Resorption: Pathologic Processes and Imaging Considerations. Semin Orthod 2013: 19: 97105.
36. Abramowicz S, Cheon JE, Kim S, Bacic J, Lee EY (2011) Magnetic Resonance Imaging of Temporomandibular Joints in Children With Arthritis. J Oral Maxillofac Surg 69: 2321-2328.

37. Cisneros GJ, Kaban LB (1984) Computerized skeletal scintigraphy for assessment of mandibular asymmetry. J Oral Maxillofac Surg 42 : 513-520.

38. Pogrel MA, Kopf J, Dodson TB, Hattner R, Kaban LB (1995) A comparison of single-photon emission computed tomography and planar imaging for quantitative skeletal scintigraphy of the mandibular condyle. Oral Surg Oral Med Oral Pathol Oral Radiol 80: 226-231.

39. Pripatnanont $P$, Vittayakittipong $P$, Markmanee $U$, Thongmak $S$, Yipintsoi T (2005) The use of SPECT to evaluate growth cessation of the mandible in unilateral condylar hyperplasia. Int J Oral Maxillofac Surg 34: 364-368.

40. Hoppenreijs TJ, Stoelinga PJ, Grace KL, Robben CMG (1999) Longterm evaluation of patients with progressive condylar resorption following orthognathic surgery. Int J Oral Maxillofac Surg 28: 411418.

41. Merkx MAW, Van Damme PA (1994) Condylar resorption after orthognathic surgery: Evaluation of treatment in 8 patients. J Craniomaxillofac Surg 22: 53-58.

42. Gunson MJ, Arnett GW, Milam SB (2012) Pathophysiology and Pharmacologic Control of Osseous Mandibular Condylar Resorption. J Oral Maxillofac Surg 70: 1918-1934.

43. Goncalves JR, Wolford LM, Cassano DS, da Porciuncula G, Paniagua B, et al. (2013) Temporomandibular joint condylar changes following maxillomandibular advancement and articular disc repositioning. J Oral Maxillofac Surg 71: 1759.

44. Gomes LR, Cevidanes LH, Gomes MR, Ruellas AC, Ryan DP, et al. (2017) Counterclockwise maxillomandibular advancement surgery and disc repositioning: can condylar remodeling in the long-term follow-up be predicted? Int J Oral Maxillofac Surg 46: 1569-1578.

45. Xi T, Schreurs R, van Loon B, de Koning M, Berge S, et al. (2015) 3D analysis of condylar remodelling and skeletal relapse following bilateral sagittal split advancement osteotomies. J Craniomaxillofac Surg 43: 462-468.

46. Xi T, van Luijn R, Baan F, Schreurs R, de Koning $M$, et al. (2017) Three-dimensional analysis of condylar remodeling and skeletal relapse following bimaxillary surgery: A 2-year follow-up study. J Craniomaxillofac Surg 45: 1311-1318.

47. Yin Q, Abotaleb B, Bi R, Zhu S (2020) The quantitative correlation between condylar resorption and skeletal relapse following mandibular advancement in skeletal class II malocclusion patients. J Craniomaxillofac Surg 48: 839-844.

48. Claus JDP, Koerich L, Weissheimer A, Almeida MS, de Oliveira RB (2019) Assessment of condylar changes after orthognathic surgery using computed tomography regional superimposition. Int J Oral Maxillofac Surg 48: 1201-1208.

49. Bianchi J, Porciúncula GM, Koerich L, Ignácio J, Wolford LM, et al. (2018) Three-dimensional stability analysis of maxillomandibular advancement surgery with and without articular disc repositioning. J Craniomaxillofac Surg 46: 1348-1354.

50. Park JH, Park JJ, Papademetriou M, Suri S (2019) Anterior open bite due to idiopathic condylar resorption during orthodontic retention of a Class II Division 1 malocclusion. Am J Orthod Dentofacial Orthop 156: 555-565. 
51. Lee GH, Park JH, Lee SM, Moon DN (2019) Orthodontic Treatment Protocols for Patients with Idiopathic Condylar Resorption. J Clin Pediatr Dent 43: 292-303.

52. Kobayashi T, Izumi N, Kojima T, Sakagami N, Saito I, et al. (2012) Progressive condylar resorption after mandibular advancement. $\mathrm{Br} J$ Oral Maxillofac Surg 50: 176-180.

53. Xi T, de Koning M, Bergé S, Hoppenreijs T, Maal T (2015) The role of mandibular proximal segment rotations on skeletal relapse and condylar remodelling following bilateral sagittal split advancement osteotomies. J Craniomaxillofac Surg 43: 1716-1722.

54. Gomes LR, Cevidanes LHS, Gomes MR, de Oliveira Ruellas AC, Ryan DPO, et al. (2018) Three-dimensional quantitative assessment of surgical stability and condylar displacement changes after counterclockwise maxillomandibular advancement surgery: Effect of simultaneous articular disc repositioning. Am J Orthod Dentofacial Orthop 154: 221-233. 